\title{
Multi-channel waveform clustering: a first look at microseismic multiplets from coalbed methane stimulation
}

\author{
Tepy Septyana ${ }^{1 *} \mathbb{B}$, Andri Dian Nugraha², Sri Widiyantoro ${ }^{2}$ and Supriyono ${ }^{3}$
}

\begin{abstract}
Interpreting microseismic events triggered by reservoir stimulation (especially hydraulic fracturing) has become a common practice to understand fracture dimension and geometry. In this area of study, the need for accuracy and resolution of microseismic data is relatively high since the object of investigation is relatively small compared to other seismological studies. Hence, a robust tool is necessary to assure the quality of microseismic event locations and support the interpretation. To achieve these primary objectives, we performed a waveform clustering workflow that analyzes all waveforms representing a microseismic event. Using this approach, we identified multiplets suggesting events that are closely located and originated from the same source mechanism. We tested the workflow on microseismic data from coalbed methane stimulation. The method increases our confident on using the dataset for interpretation especially since the monitoring survey is limited by a single borehole array with very minimal spatial coverage.
\end{abstract}

Keywords: Microseismic, Waveform clustering, Multiplet

\section{Introduction}

Non-ideal survey configurations and limited knowledge of parameters in microseismic data processing manifest some degrees of uncertainty in microseismic hypocenter location. While the intention is to capture and sample seismic waves properly, most microseismic experiments are conducted in reasonably practicable operations. Determining microseismic source location is also factored by the lack of information about the medium in which seismic waves travel. Additionally, for most routine workflows that use travel-time inversion method, the resulted source locations are driven by the uncertainty in travel-time information extracted from seismograms.

In an attempt to obtain reliable results and meaningful interpretation from microseismic data, waveform clustering has been used for improving the solution of source

*Correspondence: tepy.itb2013@gmail.com

${ }^{1}$ Geophysical Engineering Study Program, Faculty of Mining

and Petroleum Engineering, Institut Teknologi Bandung, Bandung 40132,

Indonesia

Full list of author information is available at the end of the article information. This owes to earlier findings in seismology that repeating earthquake events originating from the same fault plane will have similar waveforms (Geller and Mueller 1980). In other words, those similar events have similar source mechanism and propagate through the medium with identical properties. A group of similar events is referred to a multiplet or specifically a doublet for two similar earthquake events (Poupinet et al. 1984). By performing waveform clustering for multiplet identification and analysis, we can assess the reliability of source properties (hypocenter location, moment tensor, etc.) and medium properties such as velocity, anisotropy, and attenuation. (Poupinet et al. 1984; Jones et al. 2014; Castellanos and Van der Baan 2015). Thus, multiplet analysis can be a robust Quality Control (QC) tool on key parameters in routine microseismic data processing (Castellanos and Van der Baan 2015).

Application of waveform clustering has been demonstrated in various seismological studies such as seismotectonics (Poupinet et al. 1984; Orozco-alzate 2007; Adelfio et al. 2012; Nakamura et al. 2016), induced seismicity in geothermal fields (Rowe et al. 2002; Moriya 
et al. 2003; Dyer et al. 2010), oil and gas fields (Arrowsmith and Eisner 2006; De Meersman et al. 2009; Fagan et al. 2013; Jones et al. 2014), and hydraulic fracturing treatment in tight reservoirs (Castellanos and Van der Baan 2015; Kumano and Tamagawa 2016). Those studies have shown the effectiveness of waveform clustering in deducing more accurate and higher resolution subsurface information.

In general, seismic waveform clustering means grouping seismic events based on waveform similarity. Some studies used a representative recording station (Buurman et al. 2013) or a representative receiver component (Moriya et al. 2003). The choice is commonly based on signal-to-noise ratio $(\mathrm{S} / \mathrm{N})$. In this study, we simultaneously used all channels and all components representing a seismic event so that we can compare relative changes between different components and different channels. We utilized waveforms in time domain aiming to capture characteristic of $\mathrm{P}$ and $\mathrm{S}$ waves (polarity, frequency content, phase), $\mathrm{S}$ to $\mathrm{P}$ time difference, S-to-P amplitude ratio, etc.

To demonstrate our waveform clustering workflow, we evaluated a microseismic dataset from coalbed methane (CBM) stimulation, a pilot project in Indonesia. It was collected from a single array of seismic recording tool (8 channels of 3 component receivers) in a nearly vertical borehole to understand fracture behaviors generated by hydraulic fracturing. We expected that waveform clustering can identify multiplets related to fracturing coal beds. We then checked consistency within each group especially in terms of hypocenter locations. Hence, a compelling basis has been established to ensure reliability of data interpretation.

\section{Data and method}

\section{Multi-channel waveform clustering (MWC) workflow}

Microseismic event clustering begins with measuring similarity/dissimilarity of event pairs based on their waveforms. A common metric used in seismology is cross-correlation coefficient of two seismograms from two different events (Arrowsmith and Eisner 2006; De Meersman et al. 2009; Jones et al. 2014; Castellanos and Van der Baan 2015; Kumano and Tamagawa 2016). Cross-correlation coefficient is computed and normalized either in time or frequency domain. The similarity metric can also be determined based on the averaged coherency calculated from normalized cross-spectrum of event pairs (Poupinet et al. 1984; Moriya et al. 2003). Alternatively, one can also simply measure dissimilarity of two waveforms either in time or frequency domain. Using the measured dissimilarity based on normalized spectra of the waveforms has been demonstrated in some published studies (Orozco-Alzate 2007; Fagan et al. 2013).

Finding clusters based on waveform similarity metric can be performed by various workflows. Once similarity metric is determined, clustering can be done by applying a threshold. Event pairs that exceed the similarity threshold will form doublets (Aster and Scott 1993; Moriya et al. 2003; Arrowsmith and Eisner 2006; De Meersman et al. 2009; Jones et al. 2014; Castellanos and Van der Baan 2015). Arrowsmith and Eisner (2006) described a two-step process by first assigning events into doublets followed by grouping doublets into multiplets (chainlike clustering). Alternatively, a group of seismic events can only form multiplets if all pairs within the group meet the threshold criteria, and in fact are strongly correlated (Castellanos and Van der Baan 2015). No formulation exists on how a threshold is defined; the goal is to balance between clustering objectives and data quality. Low $\mathrm{S} / \mathrm{N}$ data generally require low threshold, whereas a relatively high threshold is needed to differentiate multiplets (De Meersman et al. 2009). Castellanos and Van der Baan (2015) apply a cross-correlation threshold of $90 \%$ to be able to group strongly similar events for a QC purpose. One can also iterate the process by applying a relatively high threshold to identify strongly related multiplets and then a relatively low threshold to form multiplet groups (De Meersman et al. 2009). The approach of using a threshold is also used for another similarity metric other than cross-correlation. Moriya et al. (2003) defined multiplets as any group of seismic events having an average coherency above 0.68 within a certain frequency band.

In this study, we referred multi-channel waveform clustering (MWC) as the workflow we used to identify multiplets from a set of multi-component receivers. This consists of several steps: (1) precondition the waveforms, (2) concatenate the preconditioned waveforms from three components, (3) calculate a metric of waveform dissimilarity from pairs of seismic events for each channel, (4) obtain a multi-channel dissimilarity matrix, and (5) perform clustering based on a dissimilarity matrix.

Data preconditioning prior to waveform clustering can involve filtering, normalization, and global waveform cross-correlation. A band-pass filtering is commonly adequate to remove unwanted signals such as low- and high-frequency noise but retain some characters that are useful for clustering. Then, data normalization is performed to compensate variability of energy level from different events. In a multi-component experiment, each channel can be regarded as a vector. Its normalization is based on the highest hypotenuse value on the signal coda. Lastly, time alignment is useful to optimize dissimilarity metric calculation of time-series data. Very poor 
time alignment can lead to pitfall when comparing the waveforms.

The second step is simply to concatenate the waveforms from the three components $(\mathrm{X}-\mathrm{Y}-\mathrm{Z})$ in each channel. By implementing this, we expect to have good event representations that contain all phases and directionality of seismic waves as recorded by three different components. We propose this approach as an alternative to averaging the similarity metric from each component (as in Arrowsmith and Eisner 2006).

In this study, we use the general Euclidian distance metric to determine event (dis)similarity based on waveforms in time domain. For each channel, the pairwise dissimilarity forms an $N(N-1) / 2$ array where $N$ is the number of microseismic events being studied. For every $n$ sample of time domain data, dissimilarity $d_{i j}$ is defined from a pair of microseismic events $i$ and $j$ with waveforms of $x_{i t}$ and $x_{j t}$, respectively. Since we concatenate the three component signals, it is reflected here that total samples being evaluated is $3 n$.

$$
d_{i j, c}=\left[\sum_{t=1}^{3 n}\left(x_{i t}-x_{j t}\right)^{2}\right]^{1 / 2} .
$$

As dissimilarity measures are obtained for each channel $c$, they can be combined to represent dissimilarity for all channels. Here we use the quadratic sum of dissimilarity from each individual channel.

$$
D_{i j}=\sum_{c=1}^{m} d_{i j, c}^{2} .
$$

Waveform dissimilarity can be easily visualized using a dissimilarity matrix $\mathrm{DM}_{i, j}$ which is a symmetrical $N \times N$ matrix. Each element $(i, j)$ contains waveform dissimilarity metric $D_{i j}$ between seismic event $i$ and $j$. Its diagonal element is equal to zero since each event is compared to itself (reflexivity). It also satisfies commutativity property that $D_{i, j}=D_{j, i}$.

$$
\mathrm{DM}_{i, j}=\left(\begin{array}{cccc}
D_{1,1} & D_{1,2} & \ldots & D_{1, N} \\
D_{2,1} & D_{2,2} & \cdots & D_{2, N} \\
\vdots & \vdots & \ddots & \vdots \\
D_{N, 1} & D_{N, 2} & \ldots & D_{N, N}
\end{array}\right) .
$$

Once the multi-channel dissimilarity matrix is formed, we perform clustering process using hierarchical agglomerative clustering (HAC). We choose this method for its illustrative advantages in understanding the nature of clustering from the data (Fagan et al. 2013). This method takes the dissimilarity metric to design data cluster in a hierarchical fashion. It starts by assigning each event as a cluster. Each cluster is then recursively merged with another cluster based on the dissimilarity metric. The mechanism of linkage is visualized by a hierarchical tree (i.e., a dendrogram) that allows us to design and choose criterion for data clustering.

\section{Multiplet identification and analysis from CBM microseismic data}

The field data we used to implement the multi-channel waveform clustering technique is microseismic data which recorded during stimulation phases of CBM reservoirs in Sanga-Sanga, Kalimantan. Halinda et al. (2013) presented the results and discussed the operational challenges regarding the monitoring program. The dataset was acquired by a single geophone array deployed in a nearly vertical well at around $100 \mathrm{~m}$ away from the treatment well. The array was 8 channels of 3-component receivers (one vertical and two horizontal sensors). Each channel was separated with a spacing of $30 \mathrm{~m}$ in measured depth along the wellbore. From the shallowest receiver (channel no 1), positioned at depth of $508 \mathrm{~m}$, the array spread over $210 \mathrm{~m}$ along the wellbore to the deepest receiver (channel no 8). The recorded data were sampled at $4 \mathrm{kHz}$ and stored as continuous digital signals.

The main processing workflow employed the gridsearch method based on P- and S-arrival time picks where a velocity model was built from a sonic log available at the monitoring well. Since the sensor coverage is limited spatially, hypocenter location determination utilized particle motion analysis to obtain azimuth information between microseismic sources and receivers. Figure 1a shows microseismic events located during $6 \mathrm{~h}$ of monitoring, indicating some seismic activities triggered by the stimulation program. The located microseismic events are mainly trending along the NW-SE orientation. It also appears to branch out almost orthogonally to the main fracture orientation. These apparent orientations can be interpreted as a manifestation of stress regime around the area being stimulated by injection. From both spatial and temporal analyses, we interpreted the NE-SW branches occurred later as the rock was further deformed by the stimulation.

It is highly expected that microseismic events can provide useful information to evaluate the outcome and performance of well treatment. Therefore, we used multiplet identification and analysis using the proposed waveform clustering workflow to assess the reliability of the estimated microseismic locations, so we can comfortably make an interpretation.

As described above, the first thing in the workflow was preconditioning the waveform from all events so they can be optimally used for clustering. We filtered the signals using band-pass filter with corner frequencies of 60 and 


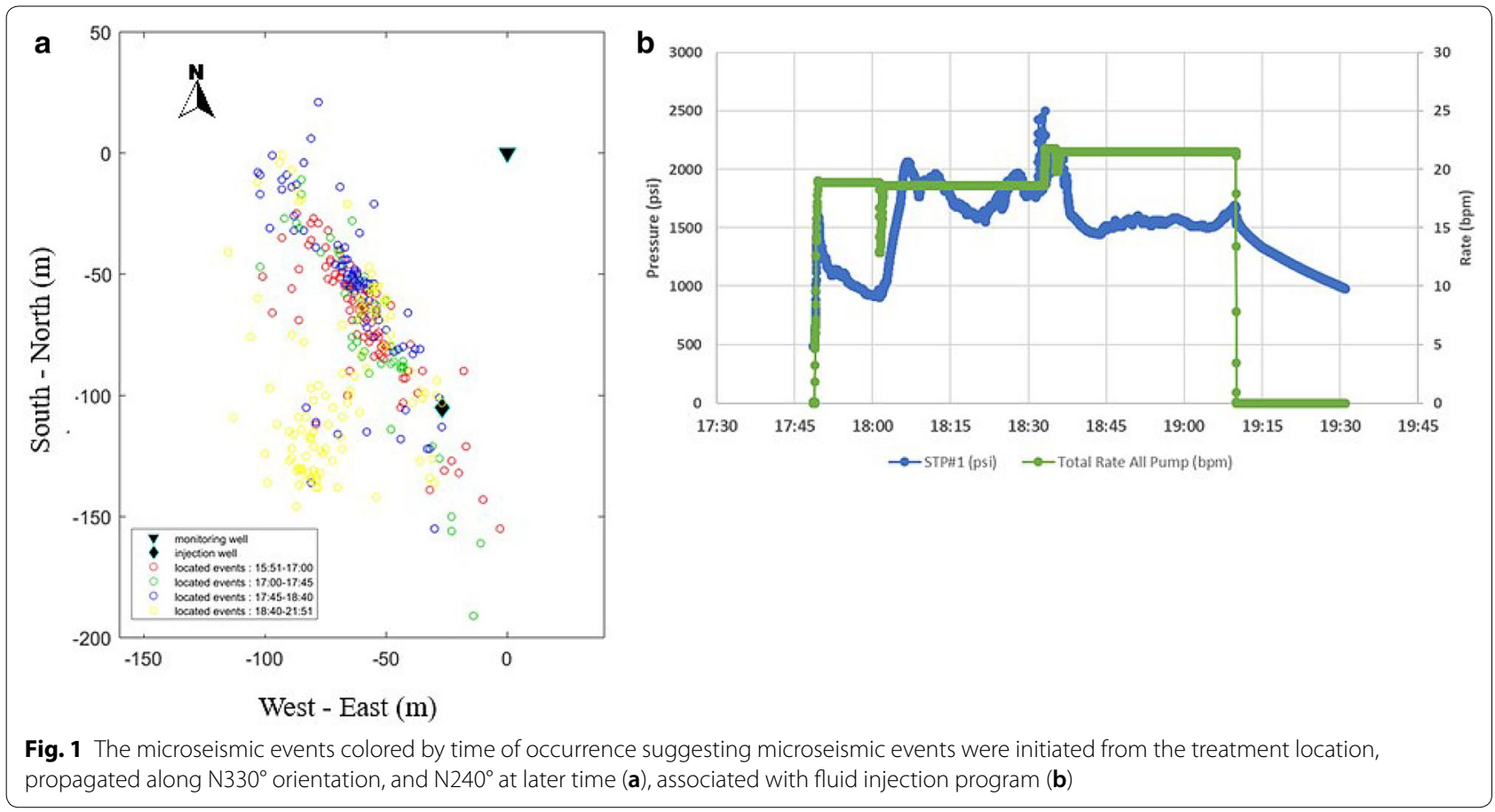

$550 \mathrm{~Hz}$ to retain useful signals for clustering, and then normalized the filtered signals to the maximum energy in each channel (based on all 3 components). Therefore, signals from different events were equalized for clustering purpose. What important in this clustering workflow is the shape of the waveforms that may represent the source mechanism or ray path of the event.

Another key ingredient in data preconditioning was to get a decent time alignment between event pairs so that the dissimilarity measure for the two events is not mistaken due to poor time alignment. We used waveform cross-correlation (WCC) technique to estimate time lags around $\mathrm{P}$ arrival time from a pair of events. The analysis was only performed on event pairs with cross-correlation coefficient greater than or equal 0.7 to ensure reliability of estimated time lags. In every calculation, an event with high $\mathrm{S} / \mathrm{N}$ was chosen as a master or reference event. Figure 2 shows waveforms before and after waveform cross-correlation. In this example, the waveform crosscorrelation technique has successfully improved time alignment with a reference of the P-wave arrival pick.

Once we are satisfied with the data preconditioning, we tested the waveform clustering workflow on 159 microseismic events, selected from the $6 \mathrm{~h}$ of monitoring. Dissimilarity metric was calculated from $500 \mathrm{~ms}$ data starting from $40 \mathrm{~ms}$ before $\mathrm{P}$ wave to encapsulate all seismic waves recorded in each event. As described in the workflow section, the calculation of waveform dissimilarity for every microseismic event pairs was done channel by channel. After dissimilarity measures were obtained per each channel, they were combined to obtain the multi-channel dissimilarity measures. This approach of calculating waveform dissimilarity measures was intended to avoid calculation pitfall if there was any incorrect sensor orientation that can occur to a wellbore array. To display some of the calculated multi-channel dissimilarity measures, a Dissimilarity matrix is shown in Fig. 3a. Each element $i j$ of the matrix contains the calculated dissimilarity measures between event $i$ and $j$, or event $j$ and $i$. We can observe zero dissimilarity (red color) in all diagonal elements of the matrix as all events are compared to itself. In contrast, brighter color towards white suggested two event pairs have high degree of dissimilarity (less or not similar). The dissimilarity matrix in Fig. 3a which is in chronological order can be reordered based on resulted clusters, in this case multiplet groups, as shown in Fig. 3b.

The clustering technique used in this study is hierarchical agglomerative clustering (HAC). The agglomerative mechanism for all 159 events can be visualized by a dendrogram (Fig. 4). All events are initialized as a single skeleton to form a cluster. A cluster is then linked to another cluster based on the dissimilarity measures which can be generalized as dissimilarity between a subset when it has more than one event. This linkage can continue until all events ultimately merge to one cluster. However, we can stop the agglomeration by setting a dissimilarity cutoff. The clusters (or 'multiplet groups') are defined as any 


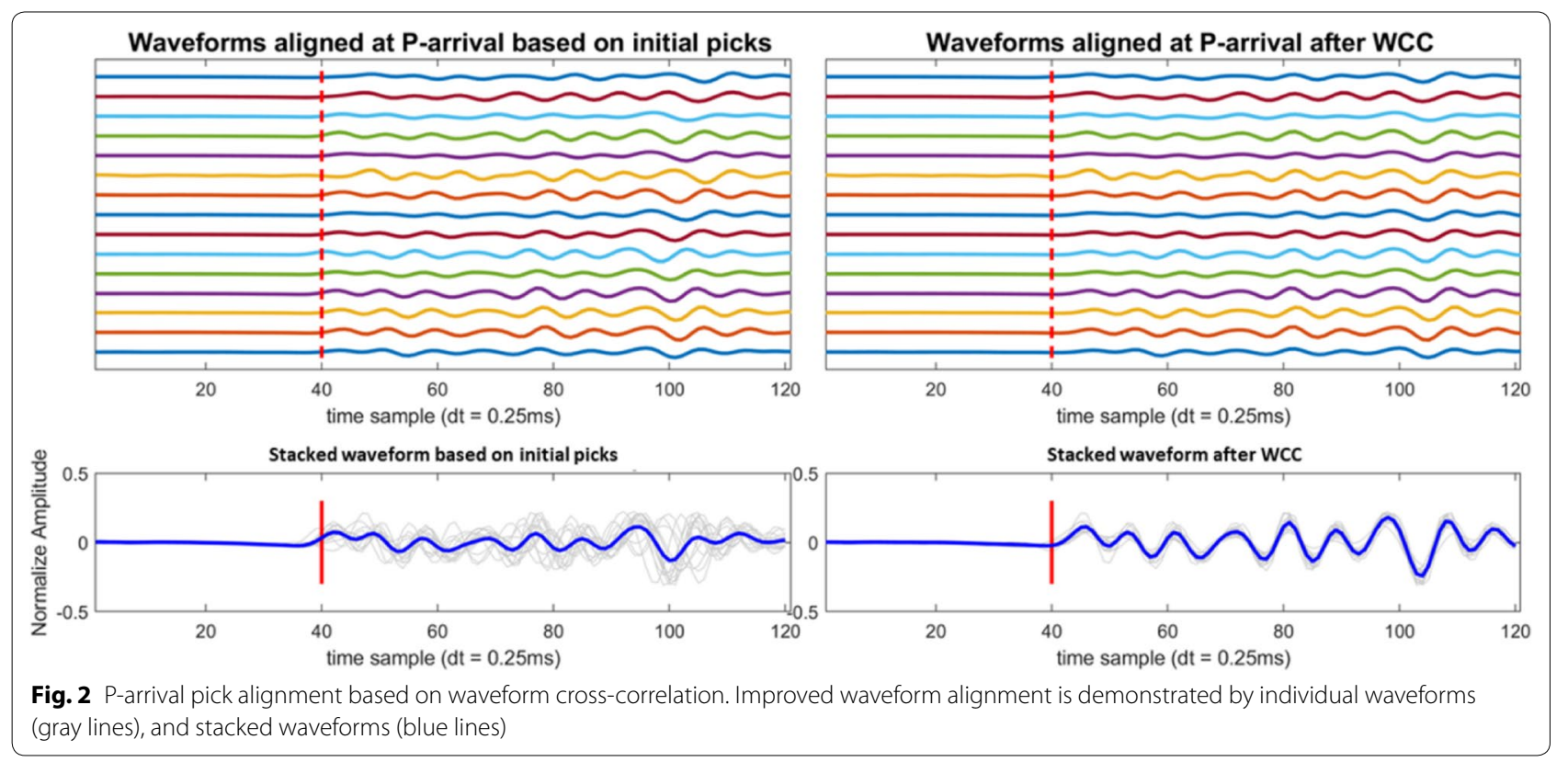

skeletons, pairs, or groups of events that are linked before exceeding the cut-off. For example, using a dissimilarity cut-off of 0.4 , we will obtain several events linked to form clusters including 3 multiplet groups with more than 4 members of highlighted microseismic events (Fig. 4). If we use 0.5 cut-off instead, bigger multiplet groups will be formed. As the cut-off increases, the total number of multiplet groups decreases, since more events or clusters can link together creating bigger-size clusters before exceeding the cut-off. The choice of cut-off can be subjectively driven by specific purposes. One can make several different cut-offs for different purposes of analysis.

\section{Results and discussion}

Using a dissimilarity cut-off of 0.4 , we discovered that $50 \%$ of the 159 events being studied are multiplets. Half of those multiplets belong to any group with 4 or more event members, and around 30\% of them are doublets. The top 9 multiplet groups (with 3 or more members) are plotted in Fig. 5 and colored differently. They are distributed in 3 different areas, named area A, B, and C. There are 5 multiplets identified in area A, 3 in B, and 1 separately located in area $\mathrm{C}$. We then evaluated the location parameters of all events within each multiplet, as summarized in Table 1. It is obvious that each multiplet has very small depth range. The standard deviation of depth for each multiplet is $2.1 \mathrm{~m}$ at maximum suggesting high likelihood that seismic activities are being constrained vertically. The back azimuth spreads around $3^{\circ}$ with the standard deviation no more than $6^{\circ}$. Another statistical measure is averaged pairwise distances which evaluates the average of geographical distances among all events within a multiplet. Multiplet group B3 and C1 have relatively bigger value in this metric as also visualized on location map.

Figure 6 shows waveforms of all events in 5 multiplet groups in area A. The color of waveforms represents each multiplet group as in Table 1, and is consistent with Fig. 5. Although all multiplet groups are geographically close (within few to $10 \mathrm{~m}$ from one event to another), the MWC has successfully identified subtle changes in the waveforms. As we can observe, the classification is quite consistent, attributed to P- and S-wave energy, polarity, S-to-P energy ratio, and $\mathrm{S}$ to $\mathrm{P}$ time delay. By looking at the time of occurrences, events in one multiplet group interleave with events from another group, for example between multiplet group A1 (orange) and A3 (blue). So, we cannot explain this due to changes in medium property. A reasonable explanation is that those multiplet groups differ in waveforms because the 2 groups might relate to different fractures. Similar waveform comparison is done for multiplet groups in areas B and C (Fig. 7). It is obvious that multiplet group $\mathrm{C} 1$ (magenta) has completely different waveforms from all other events,

(See figure on next page.)

Fig. 3 Dissimilarity matrix of microseismic event pairs sorted by chronological order (a), and reordered following the clustering result (b) 
a

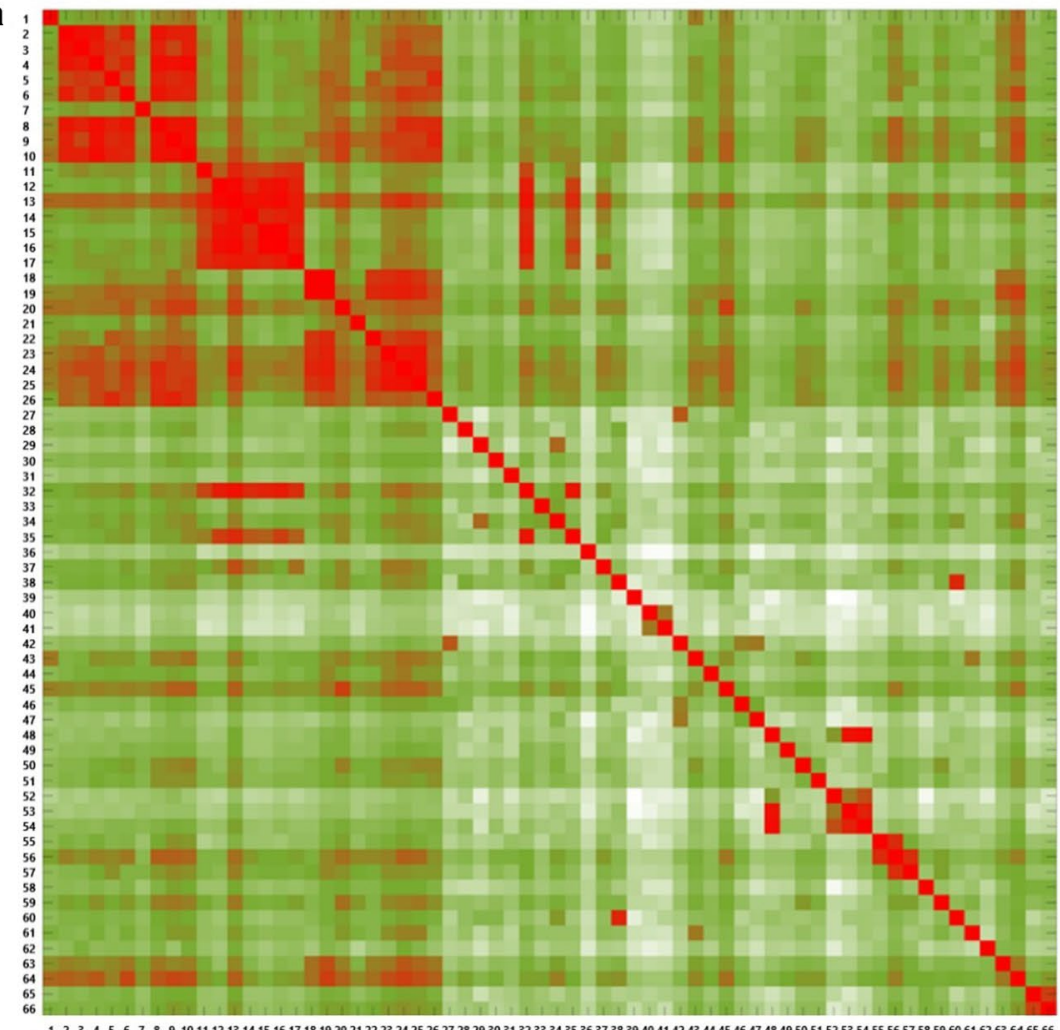

b

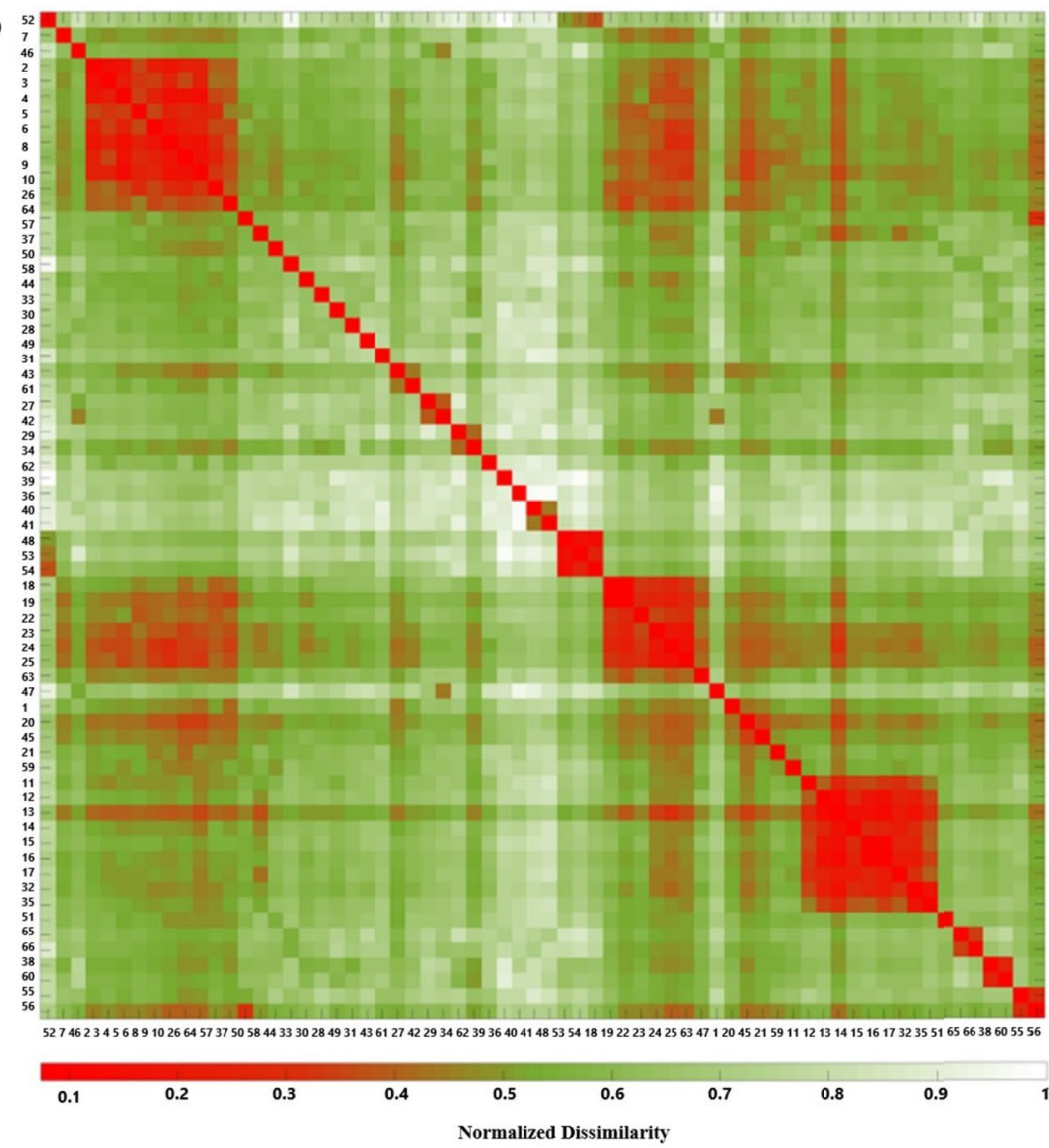




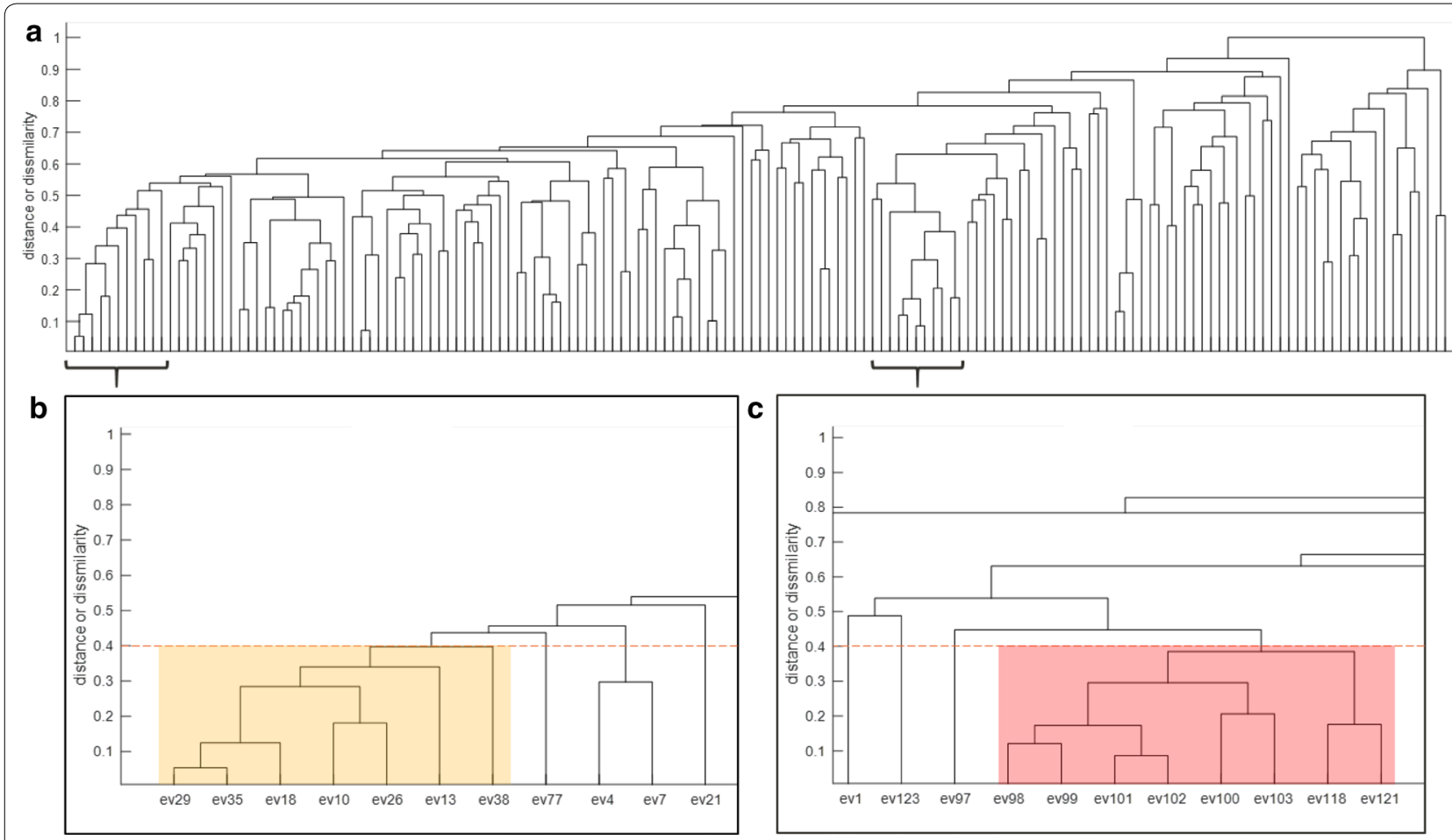

Fig. 4 A dendrogram of 159 events after hierarchical clustering (a), a basis of multiplet grouping, for example multiplet group A1 (b), and multiplet group B3 (c) are formed using a dissimilarity cut-off 0.4

suggesting it originates from a completely different source region, not from the same fracture zone as other events.

Figure 6 also demonstrates the multi-channel approach has performed effectively compensating poor variables in any sensor point (a combination between which channel and sensor component) with better variables from other sensor points. In case of multiplet groups in area A, channel 7 component $\mathrm{Z}$ has the least energy for $\mathrm{S}$ waves. Consequently, this sensor point is not a good variable for a standalone data clustering. Since the multi-channel approach was used, this is not an issue as all information from all channels and all waveforms are considered.

By looking at the hypocenter locations (Fig. 5) and the statistic results (Table 1) from each multiplet, we can notice reasonable consistency between the solution of hypocenter locations and waveform similarity. In overall, the hypocenter of all events within each multiplet are closely located and organized in a geologically sensible way. There are only a few potential mislocated events suggested by this study, one event in multiplet groups B3 and $\mathrm{C} 1$.

As we observed from Fig. 1a, the located microseismic events appear as a cloud of point sources with NW-SE major orientation. The main fracture system is oriented NW-SE. The dataset also suggests some fractures with nearly perpendicular orientation to the major orientation. Furthermore, the multiplet analysis in this study has identified few multiplets which are positioned parallel to each other following the major orientation of NWSE (Fig. 5). Based on their hypocenter locations, time of occurrence, and waveforms characteristic that we demonstrated earlier (Fig. 6), we can be confident that those different multiplets originated from different fractures. It suggests that the microseismic cloud represents a fracture network that contains many small fractures. Looking at the located hypocentres, and time of occurrence of each multiplet as well as between different multiplets, we can infer how fractures have been created, and then propagated as the stimulation progress.

\section{Conclusions}

In this paper, a workflow to identify seismic multiplets based on waveform similarity has been presented. By incorporating all waveforms from all channels and all components of the recorded events, the method is proven as a robust tool in identifying multiplets from a microseismic cloud. The grouping criterion gives reasonably confident results as illustrated by the waveform plots. Since the input is essentially the recorded waveforms, we completely avoid pitfall from incorrect assumptions such as velocity model. Therefore, it is more 

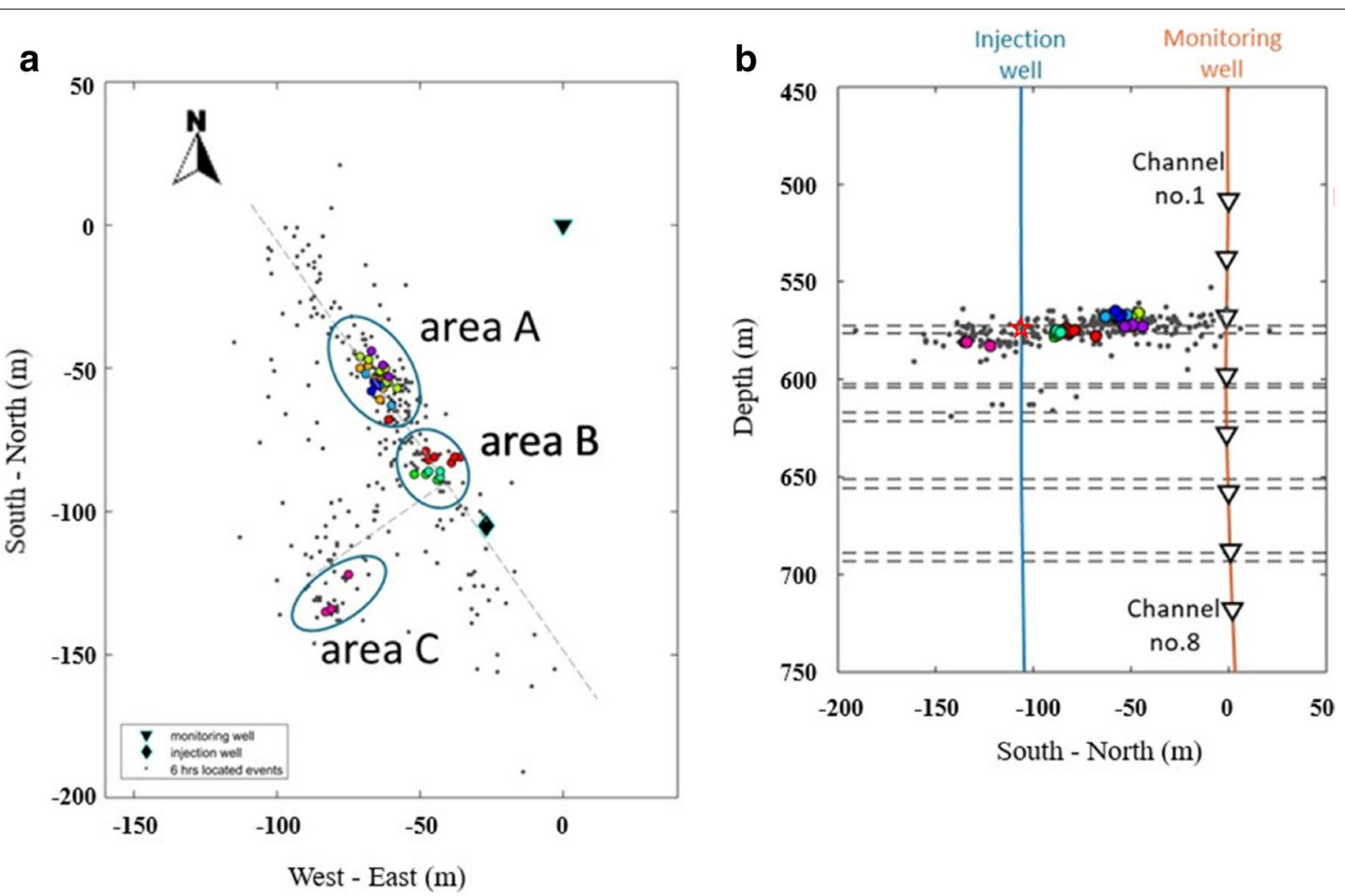

Fig. 5 Identified multiplet groups in a map view suggesting two orientations for hydraulic fracturing: primarily N330 and secondarily N240 (a). A vertical section in South-North projection showing the triggered microseismic events is mostly located in the coal zone of injection indicated by red star (b). Horizontal dash lines are coal thickness at the injection well

Table 1 Identified multiplet groups (excluding doublets), depths, back azimuth, and mean of geographical distances

\begin{tabular}{|c|c|c|c|c|c|c|}
\hline Area & Multiplet group & Color & $\begin{array}{l}\text { Number } \\
\text { of events }\end{array}$ & $\begin{array}{l}\text { Depth }(m) \\
\text { Mean } \pm \text { std. dev. }\end{array}$ & $\begin{array}{l}\text { Back Azimuth }\left({ }^{\circ}\right) \\
\text { Mean } \pm \text { std. dev }\end{array}$ & $\begin{array}{l}\text { Averaged } \\
\text { pairwise } \\
\text { distances }(\mathrm{m})\end{array}$ \\
\hline \multirow[t]{5}{*}{ A } & 1 & Orange & 7 & $570 \pm 2.1$ & $231 \pm 3.1$ & 6.1 \\
\hline & 2 & Dark blue & 3 & $567 \pm 1.5$ & $230 \pm 0.6$ & 2.5 \\
\hline & 3 & Blue & 3 & $567 \pm 0.6$ & $229 \pm 4.8$ & 9.6 \\
\hline & 4 & Green & 6 & $567 \pm 0.8$ & $232 \pm 4.6$ & 8.2 \\
\hline & 5 & Purple & 3 & $573 \pm 0.6$ & $234 \pm 3.9$ & 7.2 \\
\hline \multirow[t]{3}{*}{ B } & 1 & Dark green & 4 & $577 \pm 0.8$ & $208 \pm 2.4$ & 5.4 \\
\hline & 2 & Bluish green & 3 & $575 \pm 0.6$ & $208 \pm 1.4$ & 3.5 \\
\hline & 3 & Red & 8 & $576 \pm 1.4$ & $210 \pm 5.8$ & 10.4 \\
\hline C & 1 & Magenta & 3 & $582 \pm 1.2$ & $212 \pm 0.3$ & 10.3 \\
\hline
\end{tabular}

effective than clustering based on hypocenter locations. In fact, this method helps to identify any questionable event locations that are not consistent with waveform similarity as discussed in this study. This study has also demonstrated that inferring the spread and trend of hypocenter locations is more prudent with the basis of multiplets because we can (1) identify mislocated events and (2) classify events based on similar source mechanism. This concept is very powerful for many studies that require relatively higher accuracy microseismic solution such as monitoring reservoir stimulation.

The MWC application to the Indonesian CBM stimulation dataset has identified several multiplets among the located microseismic events triggered by injection 


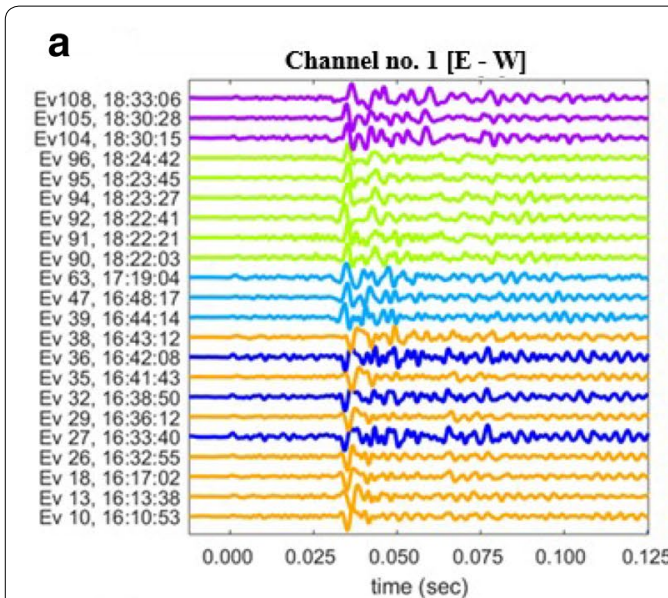

b

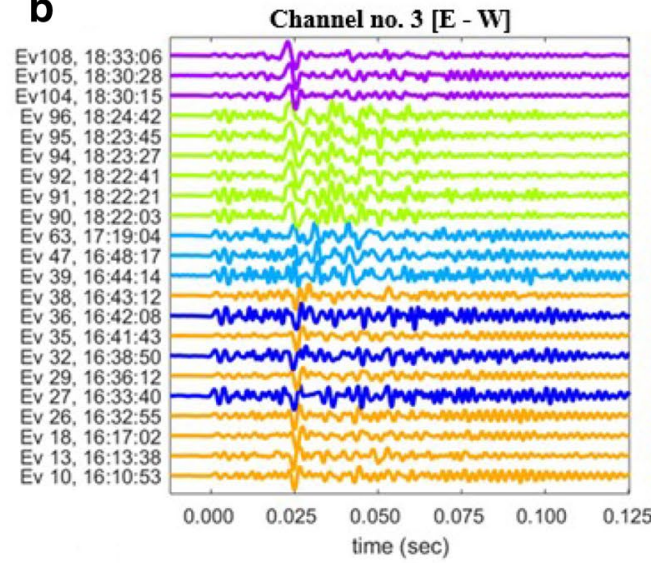

C

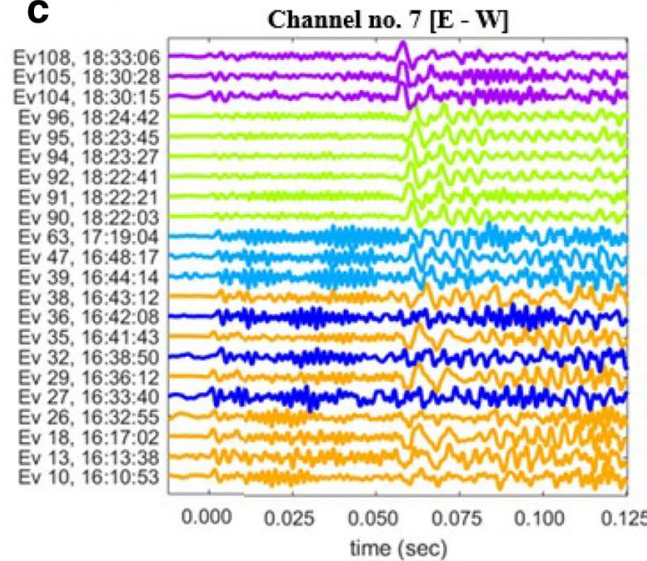

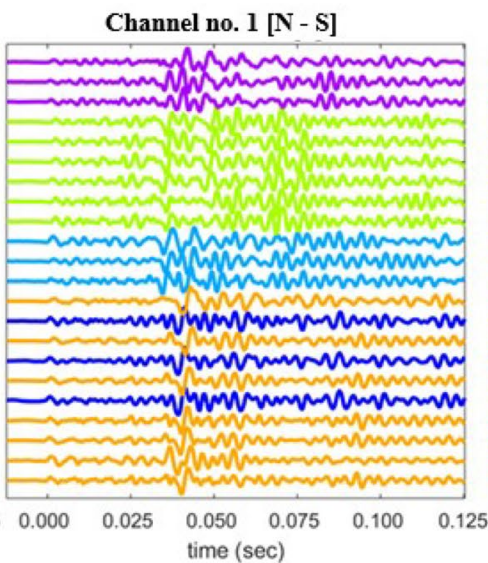

Channel no. 3 [N - S]

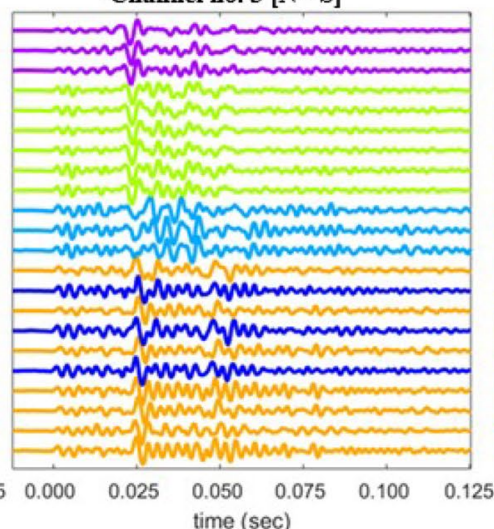

Channel no. 7 [N - S]

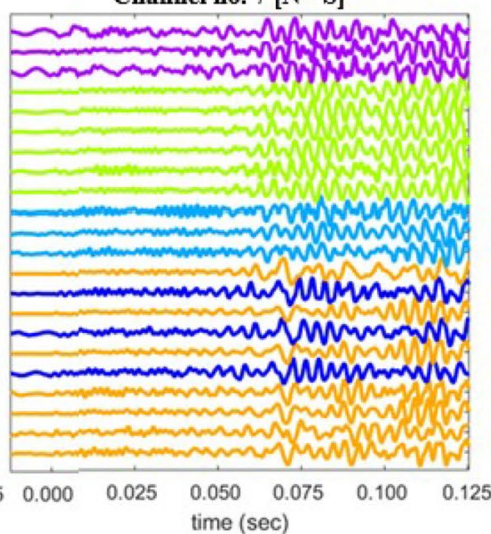

Channel no. 1 [Z]

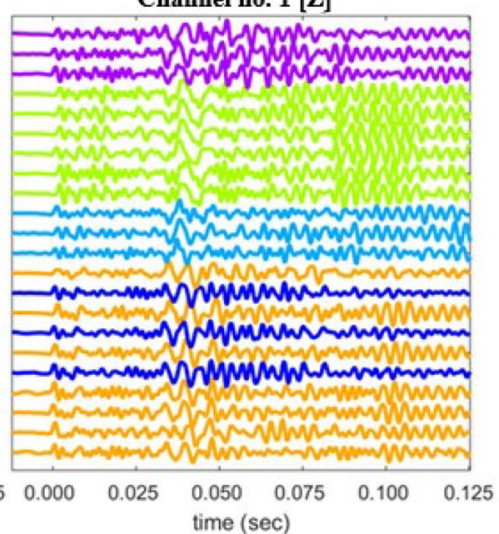

Channel no. 3 [Z]

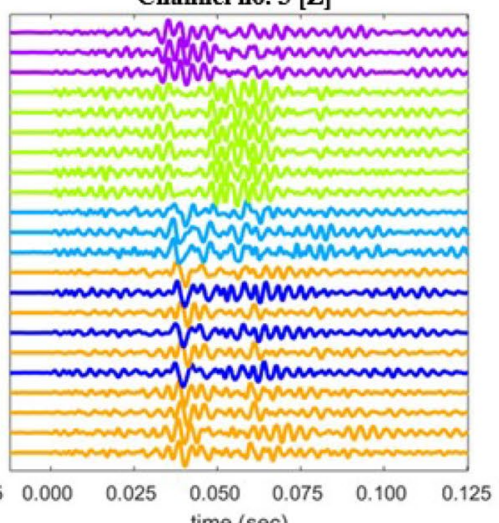

time (sec)

Channel no. 7 [Z]

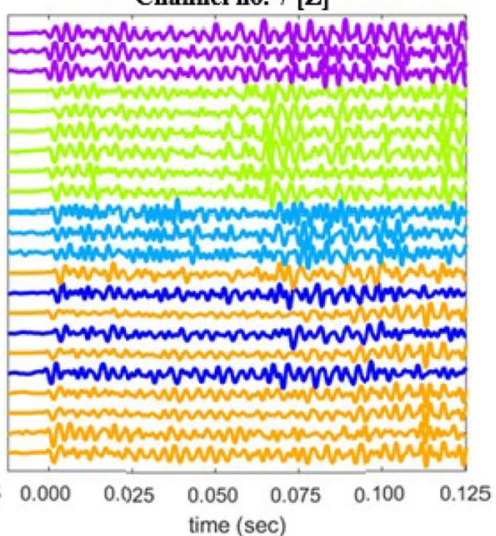

Fig. 6 Waveforms of microseismic events in area A, from three components ( 3 of 8 channels are shown) colored based on multiplet groups: A1, A2, A3, A4, A5

program. The first look and analysis at those multiplets in this study has gained more confident on the hypocenter locations. This study has also enriched the interpretation of fracture networks in the system. The spatial and temporal analysis of multiplets provides a meaningful interpretation of how fractures have been evolved in the context of injection activity. By implementing this method, we have demonstrated that we 


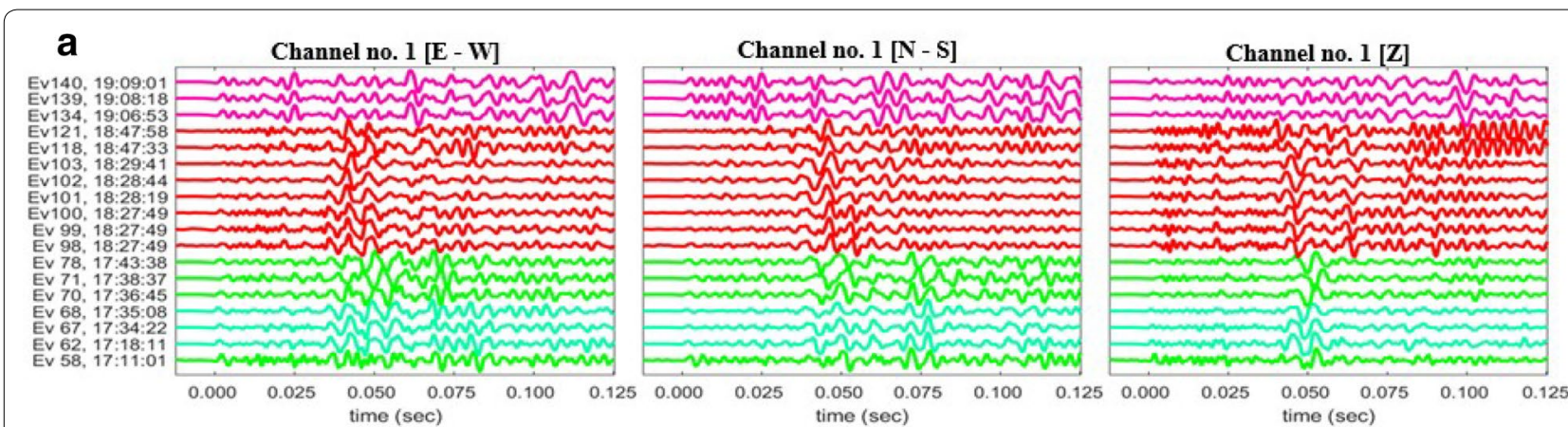

\section{b}

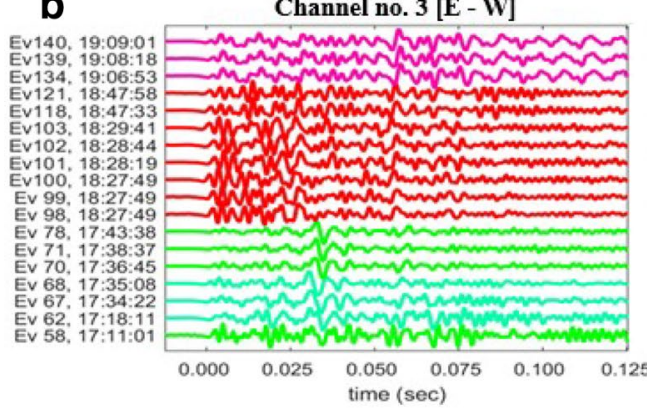

C

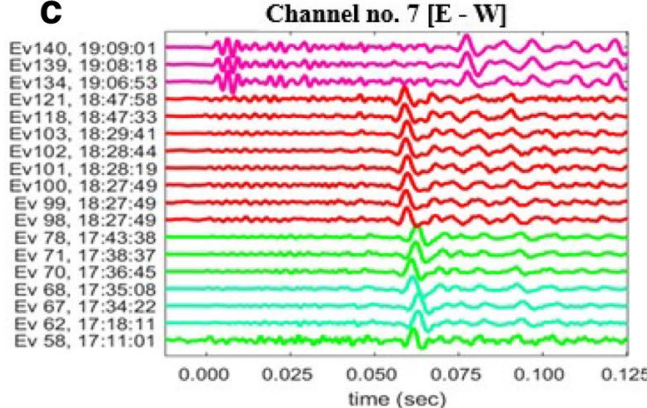

Channel no. 3 [N - S]

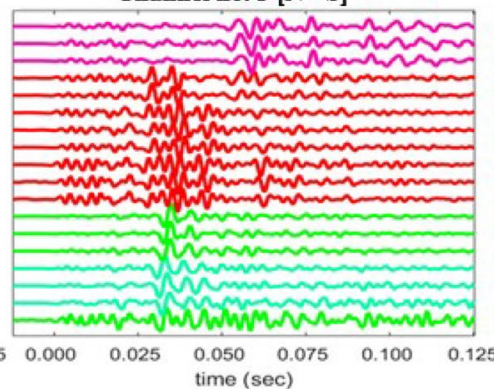

Channel no. 7 [N - S]

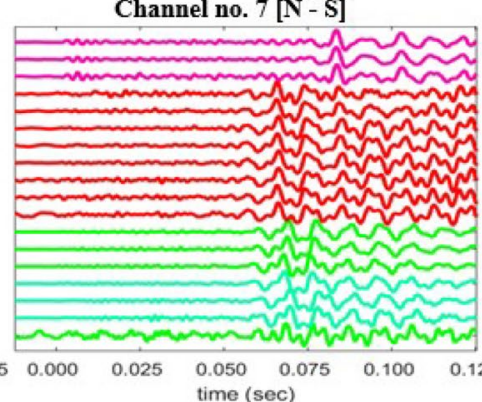

Channel no. 3 [Z]

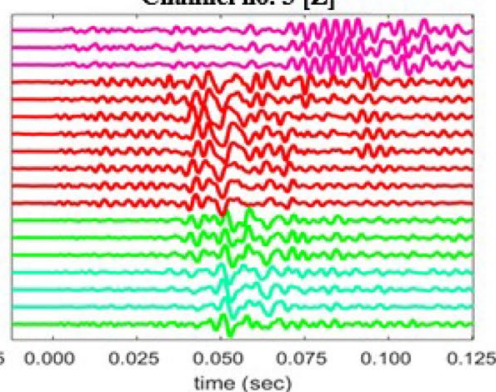

Channel no. 7 [Z]

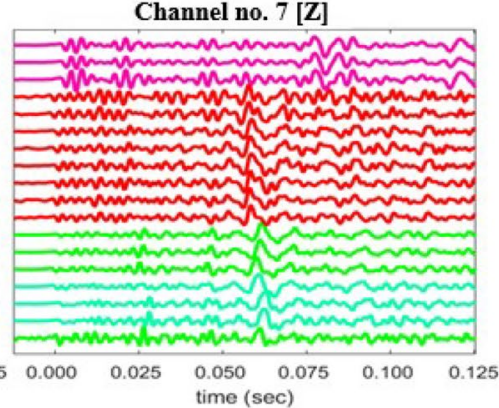

time (sec)

Fig. 7 Waveforms of microseismic events in areas B and C, from three components (3 of 8 channels are shown) colored based on multiplet groups: $B 1, B 2, B 3, C 1$

can extend microseismic data interpretation to evaluate CBM stimulation program, from looking at fault trend in general to a more detail analysis about the fracture networks.

\section{Acknowledgements}

The author would like to thank to Ardianto, a fellow student of Geophysical Engineering Study Program, Faculty of Mining and Petroleum Engineering, Institut Teknologi Bandung for many fruitful discussions during this study.

\section{Authors' contributions}

All authors read and approved the final manuscript.

\section{Funding}

Not applicable.

\section{Availability of data and materials}

The datasets analyzed during this study are not publicly available due to the Government of Indonesia data restriction.

\section{Competing interests}

The authors declare that they have no competing interests.

\section{Author details}

${ }^{1}$ Geophysical Engineering Study Program, Faculty of Mining and Petroleum Engineering, Institut Teknologi Bandung, Bandung 40132, Indonesia. ${ }^{2}$ Global Geophysics Research Group, Faculty of Mining and Petroleum Engineering, Institut Teknologi Bandung, Bandung 40132, Indonesia. ${ }^{3}$ Independent Consultant, Bandung, Indonesia.

Received: 20 January 2019 Accepted: 22 April 2019

Published online: 11 May 2019

\section{References}

Adelfio G, Chiodi M, D'Alessandro A, Luzio D, D'Anna G, Mangano G (2012) Simultaneous seismic wave clustering and registration. Comput Geosci 44:60-69

Arrowsmith SJ, Eisner L (2006) A technique for identifying microseismic multiplets and application to the Valhall field, North Sea. Geophysics $71: \mathrm{V} 31-\mathrm{V} 40$

Aster RC, Scott J (1993) Comprehensive characterization of waveform similarity in microearthquake data sets. Bull Seismol Soc Am 83:1307-1314

Buurman H, West ME, Thompson G (2013) The seismicity of the 2009 Redoubt eruption. JVolcanol Geoth Res 259:16-30 
Castellanos F, van der Baan M (2015) Waveform similarity for quality control of event locations, time picking, and moment tensor solutions. Geophysics 80:WC99-WC106

De Meersman K, Kendall J-M, van der Baan M (2009) The 1998 Valhall microseismic data set: an integrated study of relocated sources, seismic multiplets, and S-wave splitting. Geophysics 74:B183-B195

Dyer BC, Schanz U, Spillmann T, Ladner F, Ring MOH (2010) Application of microseismic multiplet analysis to the Basel geothermal reservoir stimulation events. Geophys Prospect 58:791-807

Fagan D, van Wijk K, Rutledge J (2013) Clustering revisited: A spectral analysis of microseismic events. Geophysics 78:KS41-KS49

Geller RJ, Mueller CS (1980) Four similar earthquakes in central California. Geophys Res Lett 7:821-824

Halinda D, Sesaro AW, Weatherall GD, Nugraha H, Collecott DJ (2013) The microseismic monitoring of hydraulic fracturing treatments of a CBM through the simultaneous use of downhole monitoring tools in the stimulated and an offset well. A case study-Sanga-Sanga CBM, Mahakam Delta, East Kalimantan, Indonesia. Society of Petroleum Engineers

Jones GA, Kendall JM, Bastow ID, Raymer DG, Wuestefeld A (2014) Characterization of fractures and faults: a multi-component passive microseismic study from the Ekofisk reservoir. Geophys Prospect 62:779-796

Kumano Y, Tamagawa T (2016) Hydraulic fractures delineated by a relative microseismic multiplets hypocenter imaging technique: a case of single well monitoring at Eagle Ford shale field. Society of Petrophysicists and Well-Log Analysts
Moriya H, Niitsuma H, Baria R (2003) Multiplet-clustering analysis reveals structural details within the seismic cloud at the Soultz Geothermal Field, France. Bull Seismol Soc Am 93:1606-1620

Nakamura W, Uchida N, Matsuzawa T (2016) Spatial distribution of the faulting types of small earthquakes around the 2011 Tohoku-oki earthquake: a comprehensive search using template events. J Geophys Res $121: 2591-2607$

Orozco-alzate M (2007) Clustering on dissimilarity representations for detecting mislabelled seismic signals at Nevado del Ruiz volcano. Earth Sci Res J 11:131-138

Poupinet G, Ellsworth WL, Frechet J (1984) Monitoring velocity variations in the crust using earthquake doublets: an application to Calaveras Fault, California. J Geophys Res 89:5719-5731

Rowe C, Aster R, Phillips W, Jones R, Borchers B, Fehler M (2002) Using automated, high-precision repicking to improve delineation of microseismic structures at the Soultz geothermal reservoir. Pure Appl Geophys 159:563-596

\section{Publisher's Note}

Springer Nature remains neutral with regard to jurisdictional claims in published maps and institutional affiliations.

\section{Submit your manuscript to a SpringerOpen ${ }^{\circ}$ journal and benefit from:}

- Convenient online submission

- Rigorous peer review

- Open access: articles freely available online

- High visibility within the field

- Retaining the copyright to your article

Submit your next manuscript at $\boldsymbol{\nabla}$ springeropen.com 